\title{
Predicting response to treatment in chronic inflammatory demyelinating polyradiculoneuropathy
}

\author{
Y-C Chan, D C Allen, D Fialho, K R Mills, R A C Hughes
}

J Neurol Neurosurg Psychiatry 2006;77:1 14-1 16. doi: 10.1136/jnnp.2004.056515

\begin{abstract}
Objective: To discover whether Inflammatory Neuropathy Cause and Treatment Group (INCAT) electrophysiological criteria for demyelinating neuropathy predict response to immunotherapy in chronic inflammatory demyelinating polyradiculoneuropathy (CIDP).

Methods: This was a retrospective case note study of patients who had attended Guy's Hospital Peripheral Nerve Clinic between January 2001 and March 2004, been diagnosed as having CIDP, and given treatment with corticosteroids, intravenous immunoglobulin (IVlg), or plasma exchange (PE). Patients' nerve conduction studies (NCS) were reviewed for evidence of demyelination and whether the abnormalities fulfilled modified INCAT electrophysiological criteria. Patients whose NCS fulfilled the criteria were assigned to the neurophysiologically definite CIDP group, while those that did not were labelled as neurophysiologically probable CIDP. Responses to any of the three immunotherapy agents were compared between the two groups.

Results: Out of 50 patients, 27 (54\%) were classified as neurophysiologically definite and $23(46 \%)$ as neurophysiologically probable CIDP patients. Twenty $(74 \%)$ neurophysiologically definite and 17 (73.9\%) neurophysiologically probable CIDP patients responded to treatment.

Conclusions: INCAT electrophysiological criteria did not predict a higher rate of response to immunotherapy. Neurophysiologically probable CIDP patients should be given a trial of immunotherapy.
\end{abstract}

$\mathrm{N}$ erve conduction studies (NCS) are critical in distinguishing between demyelinating and axonal neuropathies. However, in chronic inflammatory demyelinating polyradiculoneuropathy (CIDP), the multifocal pathology, predilection for proximal nerve segments, and coexistence of demyelination and axonal degeneration make it difficult to lay down absolute criteria. Research electrophysiological criteria for demyelination were designed to exclude patients in whom conduction slowing is due to axon degeneration. ${ }^{1-3}$ Such criteria exclude CIDP patients with mild conduction slowing or significant axon degeneration. However, patients with NCS having some features of conduction slowing but not fulfilling research criteria are often encountered.

We carried out a retrospective study to discover whether patients that fulfil research electrophysiological criteria for demyelination have a higher likelihood of responding to immunotherapy.

\section{METHODS}

This was a retrospective case note study of patients who had attended Guy's Hospital Peripheral Nerve Clinic between January 2001 and March 2004, been diagnosed as having
CIDP, and given treatment with corticosteroids, intravenous immunoglobulin (IVIg), or plasma exchange (PE). All patients had a polyneuropathy with an onset that was progressive over at least 8 weeks and neurophysiological tests suggestive of demyelination. Clinical evaluation and blood investigations excluded inherited, toxic, or metabolic causes and paraproteinaemia. Most patients had cerebrospinal fluid (CSF) analysis and some, nerve biopsies or genetic testing for common mutations causing Charcot-Marie-Tooth disease. We included patients with diabetes mellitus where we considered that CIDP rather than metabolic neuropathy was the principal cause. We excluded patients with multifocal motor neuropathy and patients with monoclonal paraproteinaemia at the initial evaluation.

\section{Neurophysiology tests}

All patients had NCS carried out with surface electrodes by clinical neurophysiologists. The NCS were reviewed for electrophysiological evidence of demyelination and whether the abnormalities fulfilled modified Inflammatory Neuropathy Cause and Treatment Group (INCAT) electrophysiological criteria for demyelinating neuropathy. ${ }^{4}$ Modification of the criteria was necessary because the NCS did not always have the full recommended number of nerves, nerve segments, or measurement parameters recorded. Motor recordings from median (wrist, elbow), ulnar (wrist, below elbow, above elbow), tibial (ankle, popliteal fossa), and common peroneal (ankle, below fibular head, above fibular head) nerves were included in our analysis and sensory recordings from the median, ulnar, radial, and sural nerves. We defined a demyelination abnormality (DA) as any one of the following: reduced motor conduction velocity (CV), prolonged distal motor latency (DML), absent or prolonged F-latency in demyelinating range as defined by the INCAT criteria, or partial conduction block with or without temporal dispersion when there was at least a $40 \%$ drop in peak-topeak amplitude. The tibial nerve was excluded in consideration of conduction block/temporal dispersion. A demyelinated motor nerve was one with at least one DA.

The NCS was considered to have fulfilled INCAT criteria when either one of the following conditions was present: conduction block/temporal dispersion was present in at least two nerves and at least one other nerve had a CV, DML, or Flatency DA; or a CV, DML, or F-latency DA was present in at least three nerves.

Abbreviations: ASSM, asymmetrical sensorimotor; CIDP, chronic inflammatory demyelinating polyradiculoneuropathy; CSF, cerebrospinal fluid; CV, conduction velocity; DA, demyelination abnormality; DML, distal motor latency; DSSM, distal symmetrical sensorimotor; INCAT, Inflammatory Neuropathy Cause and Treatment Group; IVlg, intravenous immunoglobulin; MRC, Medical Research Council; NCS, nerve conduction studies; ODSS, Overall Disability Sum Score; PE, plasma exchange; ULN, upper limit of normal values 


\section{Neurophysiologically definite and} neurophysiologically probable CIDP

The patients were classified as having neurophysiologically definite or neurophysiologically probable CIDP according to whether their NCS fulfilled or did not fulfil the INCAT neurophysiological criteria, respectively.

\section{Treatment response}

The patients' responses to any of three immunotherapy agents, corticosteroids, IVIg, or PE, were assessed. Response was defined as an improvement in their Medical Research Council (MRC) sum score expanded to include the first dorsal interosseous muscle or in the Overall Disability Sum Score (ODSS) of at least one point in addition to improvement reported by patients or their care givers. ${ }^{56}$

\section{Clinical phenotypes}

The patients were described as having a symmetrical sensorimotor phenotype if they had symmetrical proximal and distal weakness as well as sensory symptoms or signs, and distal symmetrical sensorimotor (DSSM) phenotype if the weakness was distal only. Symmetrical motor weakness or sensory signs alone were described as symmetrical motor or symmetrical sensory phenotypes, respectively. The asymmetrical sensorimotor (ASSM) phenotype was defined by weakness differing by at least two MRC motor grades between contralateral limbs or one grade if one side had normal strength. Asymmetrical sensory involvement without weakness was excluded.

\section{RESULTS}

Out of 50 patients, 27 (54\%) were classified as neurophysiologically definite and $23(46 \%)$ as neurophysiologically probable CIDP patients. Twenty (74\%) neurophysiologically definite and 17 (74\%) neurophysiologically probable CIDP patients responded to treatment.

\section{Clinical and laboratory features}

There were 13 male and 14 female patients in the neurophysiologically definite CIDP patient group compared with 18 males and five females in the neurophysiologically probable group. The two groups had similar mean ages, duration of disease, MRC and ODSS scores, and clinical phenotypes (table 1).

CSF protein levels were raised in $79 \%$ of the neurophysiologically definite CIDP group and $89 \%$ of the neurophysiologically probable CIDP group. Sixteen $(59 \%)$ patients in the neurophysiologically definite group and $11(48 \%)$ patients in the neurophysiologically probable group had nerve biopsies. Nine $(56 \%)$ patients in the neurophysiologically definite group and six $(55 \%)$ patients in the neurophysiologically probable group had biopsy findings that showed either pathological endoneurial mononuclear cell infiltrates or demyelinated nerve fibres, or both features.

\section{Nerve conduction studies}

The mean numbers of motor and sensory nerves tested, and the number of patients with inexcitable motor nerves were similar in both groups (table 2 ). Twenty one $(78 \%)$ patients in the neurophysiologically definite group had absent sensory potentials compared with $16(70 \%)$ patients in the neurophysiologically probable group. The mean number of demyelinated motor nerves and the mean number of DA were significantly greater in the neurophysiologically definite group compared with the neurophysiologically probable group (table 2 ).

\section{Responses to immunotherapy}

Of the 27 patients in the neurophysiologically definite group, nine $(33 \%)$ had received all three forms of immunotherapy and seven $(26 \%)$ had received two forms of immunotherapy (corticosteroids and IVIg), while 11 (40\%) had only received one form of immunotherapy (two corticosteroids, nine IVIg) (see supplemental data, table 3(S), available from http:// www.jnnp.com/supplemental). Of the 23 patients in the neurophysiologically probable group, nine (39\%) had received all three forms of immunotherapy, six (26\%) had received two forms of immunotherapy (five received corticosteroids and IVIg, one received corticosteroids and PE), while eight $(35 \%)$ had only received one form of immunotherapy (one received corticosteroids and seven received

Table 2 Nerve conduction studies

\begin{tabular}{lll}
\hline & NP definite & NP probable \\
\hline $\mathrm{n}(\%)$ & $27(54)$ & $23(46)$ \\
Motor nerves tested, mean (range) & $5(3-8)$ & $4.3(2-8)$ \\
Sensory nerves tested, mean (range) & $4.3(2-8)$ & $3.8(1-8)$ \\
Patients with inexcitable nerves, $\mathrm{n}(\%)$ & $8(29.6)$ & $5(21.7)$ \\
Patients with absent SNAP, $\mathrm{n}(\%)$ & $21(77.8)$ & $16(69.6)^{*}$ \\
Number of demyelinated motor & $3.7(3-7)$ & $1.6(0-2)^{* *}$ \\
nerves, mean (range) & & \\
Number of demyelinated & & \\
abnormalities, mean (range) & $6.4(3-10)$ & $1.9(0-6)^{* *}$ \\
\hline NP, neurophysiologically; SNAP, sensory nerve action potentials. & ${ }^{*} \mathrm{p}=0.046, t$ test significant; ${ }^{* *} \mathrm{p}<0.001, t$ test significant. \\
\hline
\end{tabular}

Table 1 Clinical features

\begin{tabular}{|c|c|c|}
\hline & NP definite & NP probable \\
\hline $\mathrm{n}(\%)$ & $27(54)$ & $23(46)$ \\
\hline Patients who responded to treatment, $\mathrm{n}(\%)$ & $20(74)$ & $17(73.9)$ \\
\hline Male/female & $13 / 14$ & $18 / 5$ \\
\hline Mean (range) age at assessment, years & $54.2(14-81)$ & $53.7(17-76)$ \\
\hline Mean (SD) duration of disease, years & $7.7(6.8)$ & 9.89 (9) \\
\hline Mean (SD) MRC score at initiation of treatment & $57.8(10)$ & $53.7(14)$ \\
\hline Mean (SD) ODSS score at initiation of treatment & $6.3(1.8)$ & $6.1(2.9)$ \\
\hline Symmetrical sensorimotor phenotype, $\mathrm{n}(\%)$ & $16(59.3)$ & $14(60.9)$ \\
\hline ASSM phenotype, $\mathrm{n}(\%)$ & $3(11.1)$ & $5(21.7)$ \\
\hline DSSM phenotype, n (\%) & $5(18.5)$ & $4(17.4)$ \\
\hline Symmetrical motor phenotype, n (\%) & 1 (3.7) & 0 \\
\hline Symmetrical sensory phenotype, $n(\%)$ & $2(7.4)$ & 0 \\
\hline Patients who had CSF analysis, $\mathrm{n}(\%)$ & $24(88.9)$ & $18(78.3)$ \\
\hline Patients with CSF protein $>400 \mathrm{mg} / \mathrm{l}, \mathrm{n}(\%)$ & 19 (79.2) & $16(88.9)$ \\
\hline Patients who had nerve biopsy, n (\%) & $16(59.3)$ & $11(47.8)$ \\
\hline Patients who had pathological lymphocytic endoneurial infiltrates, $n$ & 5 & 0 \\
\hline Patients who had pathological numbers of demyelinated/remyelinated fibres, $\mathrm{n}$ & 7 & 6 \\
\hline
\end{tabular}


IVIg). All except two non-responders received a trial of at least two forms of treatment. The two exceptions did not respond to treatment with IVIg but did not receive corticosteroids.

\section{DISCUSSION}

Regardless of whether they fulfilled the INCAT neurophysiological criteria, $74 \%$ of our patients responded to immunotherapy. Although the NCS of the patients in the neurophysiologically definite group showed more demyelinated motor nerves and DA, these did not predict a higher rate of response to immunotherapy.

The criteria were poorly sensitive in identifying treatable patients: they identified $20(54 \%)$ of the patients who responded to treatment. Had we restricted treatment only to those that fulfilled the criteria, $17(46 \%)$ of the treatable patients would have been left untreated. We also analysed our data as regards the sensitivity of three other neurophysiological criteria in identifying our treatable patients. Only nine $(24 \%)$ of the treatment responders had NCS that fulfilled the American Academy of Neurology (AAN) neurophysiological criteria for demyelinating neuropathy. ${ }^{7}$ Magda et al have proposed the presence of electrodiagnostic abnormalities in at least three nerves and partial conduction block or demyelinating range abnormalities in at least one nerve as minimal criteria. ${ }^{3}$ These criteria would have identified $30(81 \%)$ of our treatment responders. Van Den Bergh and colleagues ${ }^{2}$ also proposed new criteria where fulfilment of any one of the following conditions would support a diagnosis of CIDP: prolongation of DML ( $>150 \%$ above the upper limit of normal values (ULN)); slowing of motor CV $(<70 \%$ below the lower limit of normal values); prolongation of F-wave latency ( $>125 \%$ above the ULN) or abnormal temporal dispersion in two or more nerves; F-wave absence in two nerves plus abnormality in at least one other parameter in one other nerve; motor conduction block $(>30 \%$ amplitude reduction) in at least two nerves or its presence in one nerve together with one other abnormal parameter in at least one other nerve. These criteria would have identified 29 $(78 \%)$ of our treatment responders.

Comparing the characteristics of the two groups revealed similar mean ages, duration of disease, severity, phenotypes, and CSF and nerve biopsy findings. However, there chanced to be more females patients in the neurophysiologically definite group. There was also a trend towards the neurophysiologically definite group having more patients with absent sensory potentials (table 2 ).

In conclusion, our retrospective study showed that the INCAT neurophysiological criteria were poorly sensitive for predicting response to immunotherapy. It is uncertain whether a larger, prospective study using these or other neurophysiological and treatment response criteria would reach similar conclusions. Until we have clearer evidence, patients with chronic progressive or relapsing polyneuropathies and neurophysiological tests showing features suggestive of demyelination should be given a trial of immunotherapy.

\section{ACKNOWLEDGEMENTS}

We thank Mr and Mrs Chris Lazari for financial support.

\section{ELECTRONIC-DATABASE INFORMATION}

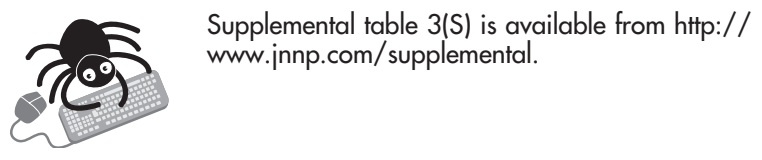

\section{Authors' affiliations}

Y-C Chan, D C Allen, D Fialho, K R Mills, R A C Hughes, Department of Clinical Neuroscience, Guy's Campus, King's College, London SEl 1UL, UK

\section{Competing interests: none declared}

Disclosures: The Department of Clinical Neuroscience has received research grants or consultancy fees from Bayer, Kedrion, LFB, and Sandoz (now Novartis). These companies manufacture or used to manufacture immunoglobulin

Correspondence to: $\operatorname{Dr}$ Y C Chan, Department of Medicine, National University Hospital, 5 Lower Kent Ridge Road, Singapore 11907, Singapore; yeecheun@yahoo.com.sg

Received 7 April 2005

Accepted 15 April 2005

\section{REFERENCES}

1 Bromberg MB. Comparison of electrodiagnostic criteria for primary demyelination in chronic polyneuropathy. Muscle Nerve 1991;14:968-76.

2 Van Den Bergh PY, Pieret F. Electrodiagnostic criteria for acute and chronic inflammatory demyelinating polyradiculoneuropathy. Muscle Nerve 2004;29:565-74.

3 Magda $\mathbf{P}$, Latov N, Brannagan TH III, et al. Comparison of electrodiagnostic abnormalities and criteria in a cohort of patients with chronic inflammatory demyelinating polyneuropathy. Arch Neurol 2003;60:1755-9

4 Hughes $R$, Bensa S, Willison $\mathrm{H}$, et al. Randomized controlled trial of intravenous immunoglobulin versus oral prednisolone in chronic inflammatory demyelinating polyradiculoneuropathy. Ann Neurol 2001;50:195-201.

5 Kleyweg RP, van der Meché FGA, Schmitz PIM. Interobserver agreement in the assessment of muscle strength and functional abilities in Guillain-Barré syndrome. Muscle Nerve 1991;14:1103-9.

6 Merkies IS, Schmitz PI, van der Meché FG, et al. Clinimetric evaluation of a new overall disability scale in immune mediated polyneuropathies. I Neurol Neurosurg Psychiatry 2002;72:596-601.

7 Ad Hoc Subcommittee of the American Academy of Neurology AIDS Task Force. Research criteria for diagnosis of chronic inflammatory demyelinating polyneuropathy (CIDP). Neurology 1991;41:617-8. 